\title{
A Survey on Circuit Modeling of Spin-Transfer- Torque Magnetic Tunnel Junctions
}

\author{
Aynaz Vatankhahghadim, Safeen Huda, and Ali Sheikholeslami
}

\begin{abstract}
Accurate modeling of magnetic tunnel junction (MTJ) is critical for design of memories such as spin-transfertorque magnetoresistive random access memory (STT-MRAM) and spin logic circuits such as spin flip flops. This paper reviews several static and dynamic models for the MTJ and compares them for their capabilities and limitations. Furthermore, a Verilog-A model is developed to predict dynamic characteristics of the MTJ. These models are used in simulating a prototype circuit to illustrate their strengths and weaknesses.
\end{abstract}

Index Terms-Magnetic tunnel junction (MTJ), magnetoresistive random-access memory (MRAM), modeling, spin-transfer-torque (STT).

\section{INTRODUCTION}

$\mathbf{S}$ PINTRONICS is an emerging field of science and engineering that uses spin of electrons, in addition to their charge, for the purpose of information storage and transport. The goal in this field is to design new devices such as spin transistors in order to improve the overall performance of electronics.

Spin-Transfer-Torque Magnetoresistive Random Access Memory (STT-MRAM), is an example of spintronic circuit, which has made significant progress in the recent years [1], [2]. STT-MRAM is a prime candidate for a universal memory because among the non-volatile memory technologies, FLASH memory (traditional non-volatile memory) suffers from low number of write endurance $\left(10^{5}\right)$ as well as high write access time $\left(10^{5}\right.$ to $\left.10^{7} \mathrm{~ns}\right)$ [3]; others such as ferroelectric memory (FeRAM) and phase-change memory (PRAM) are limited to $10^{9}$ to $10^{12}$ number of write endurance. In contrast, STT-MRAM could accommodate a high endurance (in the order of $10^{15}$ ) and a low access time (in the order of nanoseconds) [3].

Fig. 1 shows an example spintronic circuit consisting of ferromagnetic elements (with conductances $\mathrm{G}_{\mathrm{FM} 1}$ to $\mathrm{G}_{\mathrm{FM}}$ ), and non-magnetic elements (with conductances $\mathrm{G}_{\mathrm{NM} 1}$ and $\mathrm{G}_{\mathrm{NM} 2}$ ). In general, each element is characterized by a $4 \times 4$ conduction matrix which relates the element's total spin current to its total spin voltage, $\left[\mathrm{I}_{\mathrm{c}} \mathrm{I}_{\mathrm{sx}} \mathrm{I}_{\mathrm{sy}} \mathrm{I}_{\mathrm{sz}}\right]^{\mathrm{T}}=[\mathrm{G}]_{4 \times 4}\left[\mathrm{~V}_{\mathrm{c}} \mathrm{V}_{\mathrm{sx}} \mathrm{V}_{\mathrm{sy}} \mathrm{V}_{\mathrm{sz}}\right]^{\mathrm{T}}$. The total spin current has four elements, namely, the charge current, $\mathrm{I}_{\mathrm{c}}$, and the spin currents, with three Cartesian components specified by sx, sy, sz. Similarly, the total spin voltage consists of the

Manuscript received September 16, 2013; revised December 20, 2013 and January 30, 2014; accepted February 16, 2014. Date of publication July 08, 2014; date of current version August 26, 2014. This work was supported in part by NSERC. This paper was recommended by Associate Editor M. M. Khellah.

The authors are with the Department of Electrical and Computer Engineering, University of Toronto, Toronto ON M5S, Canada (e-mail: aynaz@eecg.utoronto.ca; safeen@eecg.utoronto.ca; ali@eecg.utoronto.ca).

Color versions of one or more of the figures in this paper are available online at http://ieeexplore.ieee.org.

Digital Object Identifier 10.1109/TCSI.2014.2332247

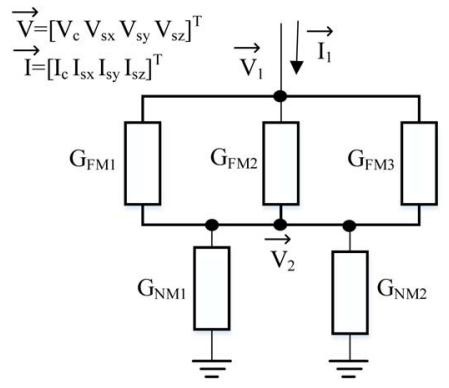

Fig. 1. Example spintronic circuit.

charge voltage $\left(\mathrm{V}_{\mathrm{c}}\right)$, and the spin voltages of $\mathrm{V}_{\mathrm{sx}}, \mathrm{V}_{\mathrm{sy}}$, and $\mathrm{V}_{\mathrm{sz}}$ [4].

Authors in [5] present a theoretical formalism for analysis and design of spintronic integrated circuits by discussing spin conduction matrices, spin current/voltage and extending modified nodal analysis. However, in a subset of spintronic circuits, such as in STT-MRAM, these conduction matrices are reduced to single conductance parameters which can be calculated from what is known as Julliere's model (which will be discussed in Section III-A). This is because the interconnect distance between spin-related devices (magnetic tunnel junction (MTJ) in the case of STT-MRAM) are larger than the spin diffusion length, which is defined as propagation distance of non-equilibrium spin population and it is in the order of 5-1000 nm [5]. As a result, the current becomes unpolarized once it leaves an MTJ and hence can be treated as charge current only. It is for this scenario that we discuss MTJ modeling in this paper.

An accurate model for the MTJ should be developed to predict the MTJ behavior in hybrid MTJ/CMOS circuits. In order to address challenges of STT-MRAM design, such as reducing the switching current [6] or dealing with process variation [7], [8] and read-disturbance issue [3], a compact MTJ model compatible with CMOS design tools such as SPICE [9] and SPECTRE [10] is necessary. On the other hand, modeling the MTJ has its own challenges such as accurately characterizing the static/dynamic behavior of the device as well as considering thermal effects, stochastic processes, and process variations.

This paper focuses on modeling the MTJ of STT-MRAM cell and presents a survey as following. Section II includes the background for MRAM cell. Section III discusses the modeling basics, categorizing the models into two groups of static and dynamic models. Section IV reviews some of the existing static models, and then in Section V these models are compared. Similarly, in Section VI dynamic models including our developed model are reviewed. Our implemented MTJ model is used for comparison of dynamic models in Section VII. This is followed by conclusion. 

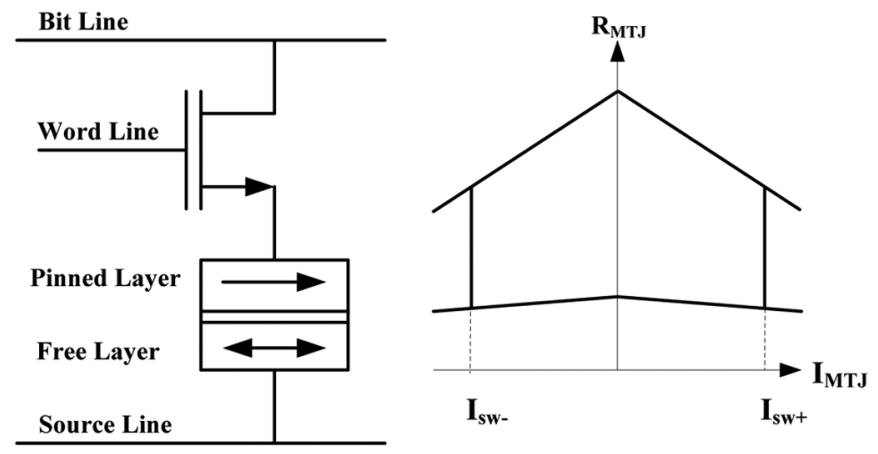

Fig. 2. Conventional STT-MRAM cell and hysteresis behavior of MTJ.

\section{BACKGROUND}

The conventional MRAM cell consists of an MTJ and an access transistor, as shown in Fig. 2. An MTJ, as the main element of the memory cell, consists of two ferromagnetic layers with a thin insulating layer in between. It includes one fixed magnetized layer and one free layer, whose magnetization can be changed by spin-polarized current in the process of writing to the memory. Depending on the direction of the current, magnetization of the free layer will be aligned either in parallel or anti-parallel to the pinned layer.

Slonczewski [11] stated that in magnetic multi-layers, spinpolarized electrons of the magnetic layer will experience some change in their angular momentum while passing from one magnetic layer to another with different magnetization vector. Because of angular momentum conservation, the difference in the initial and final angular momentum will be absorbed by the two magnetic layers, resulting in the torque applied on the magnetization vectors. This is called spin-transfer-torque.

The read operation involves measuring the effective resistance of the MTJ in two different states representing " 0 " and " 1 ". The resistance between the pinned layer and the free layer is higher when the layers are anti-parallel $\left(\mathrm{R}_{\mathrm{AP}}\right)$ than when they are parallel (Rp). This difference is represented by an MTJ parameter called Tunneling Magnetoresistance Ratio (TMR) [3] as defined by:

$$
T M R=\frac{R_{A P}-R_{p}}{R_{p}}
$$

There are two types of models describing the behavior of MTJ: static and dynamic models. Static models only describe the static characteristics of MTJ, while the dynamic models capture the dynamic behavior as well as the static properties. Static models are only concerned with whether the switching has occurred and accordingly set the MTJ resistance. On the other hand, dynamic models keep track of the magnetization vector as a function of time. As the circuits operate at higher speed, the need for dynamic models becomes inevitable.In addition, thermal effects and stochastic processes also play a role in dynamic behavior of the devices and should be taken into account for accurate modeling of the MTJ. More details on modeling basics are provided in the next section.

\section{MODELING BASICS}

\section{A. Static Modeling}

Static models predict the switching based on critical current and/or the switching time. After the decision is made on whether the switching has occurred, the resistance of the MTJ is set accordingly.

In this regard, static modeling deploys Julliere's model as in (2) to determine the MTJ conductance of its two stable states [12].

$$
G(\theta)=G_{0}\left(1+p^{2} \cos \theta\right)
$$

in which $G_{0}$ is the average conductance over $\theta, p$ is the polarization factor, and $\theta$ is the angle between the magnetization vectors of the two layers. The two states are nominally associated with $\theta=0^{\circ}$ and $\theta=180^{\circ}$. Given (2), the equation for TMR can be rewritten as TMR $=2 p^{2} /\left(1-p^{2}\right)$. This clearly shows that TMR is a direct function of $p$.

Based on the angle between the magnetization vectors, the conductance of the MTJ is set according to (2). However, Julliere's model does not capture the effects of voltage variation on conductance. Higher accuracy is attained by considering voltage-dependency of $G_{0}$ and $p$. Also, considering temperature-dependency of these parameters will further increase the accuracy.

\section{B. Dynamic Modeling}

Dynamic behavior of the magnetization vector of the magnetic materials is described by Landau-Lifshitz-Gilbert (LLG) equation as in (3) [13], [14].

$$
\begin{aligned}
\frac{\partial \vec{M}}{\partial t}=-\frac{\gamma}{1+\alpha^{2}} \vec{M} \times H_{e f f}(\theta)-\frac{\gamma \alpha}{\left(1+\alpha^{2}\right) M_{s}} \vec{M} \\
\quad \times\left(\vec{M} \times H_{e f f}(\theta)\right)
\end{aligned}
$$

Where $\gamma$ is the gyromagnetic ratio, $\alpha$ is the Gilbert damping parameter, $M_{s}$ is the saturation magnetization, $H_{e f f}$ is the effective field, and $\theta$ is the angle between the easy axis of the magnet and the magnetization vector.

The first term in (3) represents the precession while the second term represents the damping behavior of the magnetization vector. According to LLG equation, the magnetization vector precesses around the easy axis while it is pulled towards it. In equilibrium, the magnetization is aligned with the easy axis.

It was discovered in 1996 that in case of disturbance by a spin polarized current, an additional torque term will affect the dynamics of the magnetization vector [11]. The torque term, which should be added to the right side of (3), is given by,

$$
\vec{T}_{\text {spin }}=-\frac{\gamma \eta(\theta) \hbar}{2 e t M_{s}^{2} A} I .\left(\vec{M} \times\left(\vec{M} \times \vec{M}_{P L}\right)\right)
$$

where $t$ is the thickness of the free layer, $A$ is the cross sectional area, $e$ is the electron charge, $M_{s}$ is the saturation magnetization, $M_{P L}$ is the magnetization vector of the pinned layer, $I$ is the current going through the MTJ from the pinned layer to the free layer, and $\eta$ is the spin torque efficiency factor. The equation then becomes the LLGS (Landau-Lifshitz-Gilbert-Slonczewski) equation. LLGS equation forms the basis of several dynamic models that will be discussed later in this paper.

Another approach to capture the dynamic behavior of switching is to consider two switching regions based on the amplitude of the current [15]. When the applied current is lower than the critical current, switching occurs mainly due 


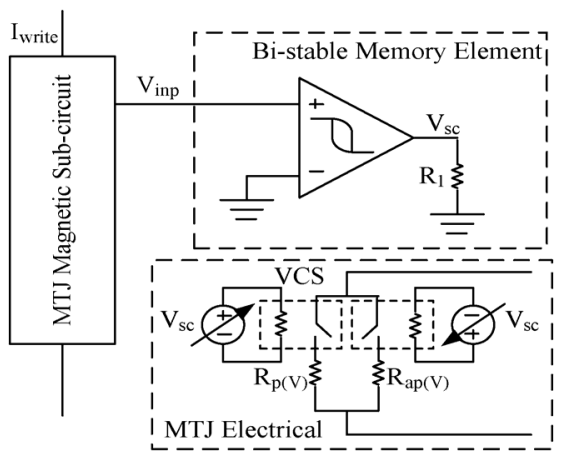

Fig. 3. Block diagram of MTJ macro-model [17].

to thermal fluctuations. In this case, Neel-Brown model [16] calculates the switching time, $\tau_{1}$, to be:

$$
\tau_{1}=\tau_{0} \exp \left(\frac{\Delta E}{k_{B} T}\left(1-\frac{I}{I_{C 0}}\right)\right)
$$

where $T$ is the temperature, $k_{B}$ is the Boltzmann constant, $1 / \tau_{0}$ is the attempt frequency, and $\Delta E$ is the energy barrier. When the applied current is higher than the critical current, Sun's model predicts the switching time to be:

$$
\tau_{2}=\frac{1}{\alpha \mu_{0} \gamma M_{s}} \cdot \frac{I_{C 0}}{I-I_{C 0}} \ln \left(\frac{\pi}{2 \theta_{0}}\right)
$$

where $\theta_{0}$ is the initial angle of magnetization vector from the easy axis, which is a random variable due to thermal fluctuation, and $\mu_{0}$ is the permeability. This approach is an approximation derived from analyzing LLG eq. for current pulses with constant amplitude. Equations (5) and (6) form the basis for some dynamic models discussed in Section VI.

Depending on the application and the required accuracy/speed of the simulation, either static models or dynamic models can be used. Static modeling is fast in terms of run time, but it does not provide the transient behavior nor the accuracy of dynamic modeling. Among dynamic modeling approaches, those utilizing (3), (4) are more accurate than those utilizing (5), (6), but require larger run time and higher computational cost due to solving LLGS differential equations. More details on these methods and their comparison will be provided in the following sections.

\section{Review of Static Models}

\section{A. Static Model 1(SM1) in SPICE}

The SPICE macro-model developed by S. S. Mukherjee et al. [17] is a static model based on the hysteresis behavior of the MTJ. It consists of three modules: magnetic module, storage module, and magnetoresistive module as shown in Fig. 3. Each module is implemented using SPICE circuit components; magnetic module converts the change in the current to corresponding voltage value according to the hysteresis characteristic of the MTJ. Storage module is a Schmitt trigger block that generates the corresponding voltage for the magnetoresistive module. Magnetoresistive module consists of two parallel branches (one for the parallel state the other for the anti-parallel one) of a voltage-controlled switch in series with a resistor. Depending on whether the layers of the MTJ are in parallel or anti-parallel configuration, the corresponding branch is activated.

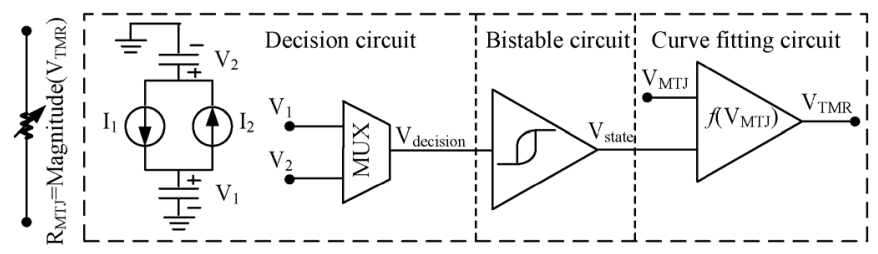

Fig. 4. Simplified block diagram of MTJ macro-model [18].

These modules along with curve-fitted functions capture the static behavior of the MTJ. However, the model is not capable of characterizing the transient behavior. Furthermore, there are no measurement results in [17] verifying the accuracy of the model.

\section{B. Static Model 2 (SM2) in SPICE}

This is a SPICE macro-model implemented by J. D. Harms et al. [18]. A simplified block diagram of the model is shown in Fig. 4. As illustrated, this model includes a decision circuit which, given a current pulse and its duration, decides whether the MTJ switches. The switching time is calculated using (5) and it is implemented exploiting the time required to charge a capacitor with a constant current. However, currents with shorter pulsewidths require higher amplitudes as thermal activation is not helpful in this case. To account for this, a fitting parameter is added to (5).

The output of the decision block enters the next block which is a bistable circuit. This circuit will provide either positive or negative voltage at the output depending on the state of the MTJ. Finally, the last block is the curve-fitting circuit which uses Gaussian functions to model the voltage across the MTJ. Some measurement results are provided in [18] to verify the accuracy of modeling the switching characteristic of the MTJ.

\section{Static Model 3 (SM3) in Verilog-A}

This is a macro-model in Verilog-A proposed by W. Zhao et al. [19]. Julliere's model is used to determine the conductance of different MTJ states. Furthermore, Brinkman's model for tunneling conductance [20] is employed to consider the voltage-dependency of the MTJ conductance. According to Brinkman's model, bias-dependent conductance is modeled as:

$$
G_{0}(V)=G_{0}\left(1+\alpha V+\beta V^{2}\right)
$$

in which $\alpha$ and $\beta$ are parameters dependent on constants such as the electron mass, Plank's constant, and the height of the barrier. To this end, $G_{0}(V)$ replaces $G_{0}$ in (2).

The voltage-dependence of TMR ratio from quantum mechanical point of view is described in [21] but it is too complex for a compact model. Therefore, a fit function as in (8) is used to characterize the bias-dependency of TMR [19]:

$$
T M R(V)=\frac{T M R(0)}{1+\frac{V^{2}}{V h^{2}}}
$$

where $T M R(0)$ is the TMR ratio for zero-bias and $V h$ is the bias voltage where $T M R(0)$ is halved. Based on the physical model of Slonczewski [11], [22], a measure of switching is the critical current at zero temperature as in,

$$
I_{C 0}=\frac{2 e \alpha M_{s} V\left(H_{K}+2 \pi M_{s}\right)}{\hbar \eta}
$$

in which $\alpha$ is the damping factor, $M_{s}$ is the saturation magnetization, $H_{K}$ is the anisotropy constant, and $\eta$ is the torque 
efficiency factor. At non zero temperatures, and for a given pulsewidth, the critical current can be found as in [15]:

$$
I_{C}=I_{C 0}\left(1-\frac{k_{B} T}{E} \ln \left(\frac{t_{p}}{\tau_{0}}\right)\right)
$$

where $k_{B}$ is the Boltzmann constant, $T$ is the temperature, $E$ is the energy barrier, $t_{p}$ is the current pulsewidth and $\tau_{0}$ is the relaxation time (switching time due to critical current at zero temperature).

Contrary to the other two models, this model uses physical equations for conductance of the MTJ rather than using curvefitted functions. Only the switching region due to thermal fluctuation is considered. No measurement results are provided.

\section{Comparative Study of Static Models}

Comparing the static models discussed in Section IV, SM1 and SM2 use SPICE components to model the MTJ behavior, while SM3 employs Verilog-A. Using only SPICE components limits the modeling capabilities. For example, using Schmitt trigger block with high gain and positive feedback may result in convergence problems. As a result, an isolated Schmitt trigger block is used to avoid input and output fluctuations. On the other hand, Verilog-A model provides possibilities to mimic variations and thermal fluctuations.

As for modeling the switching time, there are two regions of switching: one due to thermal effects, the other due to spintransfer torque. SM1 considers one region only; timing is not considered in SM1. It is assumed that the applied pulses with amplitude greater than critical current (taken from hysteresis curve of the MTJ) will flip the magnetization vector. Also for SM3, there is no discussion on switching time but it is considered indirectly by using (10). It uses critical current of (9) to decide if switching occurs and calculates the required switching current according to the pulsewidth of the applied current using (10). However, this approach does not consider switching behavior for currents greater than the critical current. On the other hand, SM2 considers the switching time by using (5). It is derived using the physics behind the MTJ. Then it adds a curvefitted term to (5) to account for longer switching times for pulses with lower pulsewidths (as they require much higher amplitudes to switch the MTJ). Therefore, both switching regions are considered by SM2.

As for modeling the voltage drop across the MTJ, SM1 uses a nonlinear voltage dependent resistor and assigns a transfer function to it by using fitting functions similar to (7). In SM2, a Gaussian curve-fitted function is assumed, and finally SM3 uses Brinkman's model following (7).

None of these static models deal with the effects of temperature on TMR ratio or other parameters of the design. Furthermore, stochastic nature of the switching is not modeled. Most importantly, all the models assume constant current amplitude, which is not usually the case in 1T-1MTJ cells.

While all these models provide simulation results, [18] compares the simulation results of SM2 with the measurement results provided in [23]. Table I summarizes the comparison of the static models discussed in this section.

\section{ReVIEW of Dynamic Models}

\section{A. Dynamic Model 1 (DM1) in Verilog-A}

The dynamic model developed by L. Faber et al. [24] improves the static model in [19] by augmenting it with the dy-
TABLE I

Comparison of Static Models

\begin{tabular}{|l|c|c|c|c|}
\hline Models & Implementation & $\begin{array}{c}\text { Switching } \\
\text { regions }\end{array}$ & $\mathrm{V}_{\text {MTJ }}$ & $\begin{array}{c}\text { Measurement } \\
\text { results }\end{array}$ \\
\hline SM1 [17] & SPICE & One & Curve-fitted & No \\
\hline SM2 [18] & SPICE & Two & Curve-fitted & Yes \\
\hline SM3 [19] & Verilog-A & One & Physical & No \\
\hline
\end{tabular}

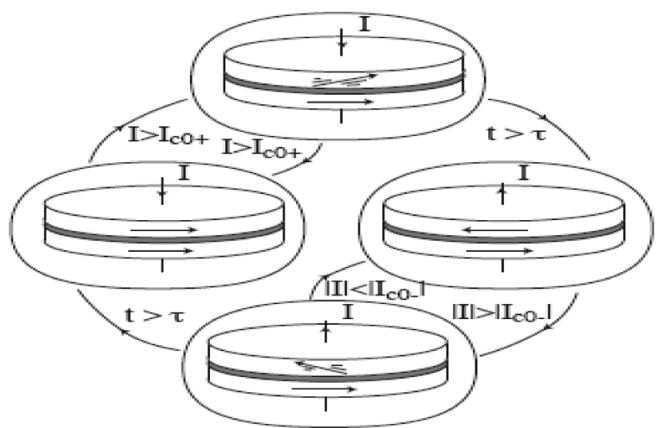

Fig. 5. Finite state machine of MTJ model [24].

namic behavior modeling. Furthermore, it incorporates heating effects and accounts for stochastic behavior due to thermal fluctuation.

Dynamic modeling is based on equations (5), (6), which consider two switching regions. It follows four computational steps starting with calculating the critical switching current using Slonczewski's model as in (9). Second step considers the cases with applied current less than critical current and uses (5) to calculate the required time for switching due to thermal fluctuations. Switching probability is described by:

$$
\operatorname{Pr}(t)=1-\exp \left(-t / \tau_{1}\right)
$$

It should be noted that the dynamic behavior of STT-MRAM is intrinsically stochastic and Monte-Carlo simulation cannot be used as it is only good for static parameter variation. Therefore, functions of random variables are combined with the model to account for the stochastic behavior.

In the third step, for the cases with currents higher than critical current, Sun's model is used as in (6). Finite state machine describing these steps is shown in Fig. 5.

The last step is considering the heating effects on behavior of STT-MRAM. Temperature rise and fall is calculated based on the voltage across the MTJ, duration of the current pulses, and thermal conductivity.

In summary, both switching regions are considered and switching time is calculated accordingly using the corresponding models and formulas. Also, thermal fluctuations are included in the analysis.

\section{B. Dynamic Model 2 (DM2) in VHDL-AMS}

The next dynamic model is the model implemented by $\mathrm{M}$. Madec et al. [25]. It is developed using VHDL-AMS. In terms of static modeling, like the model in [19], it uses the equations for $G(V)$ and $T M R(V)$ (as in (7) and (8)) along with Julliere's model for conductance. Having the conductance, which is dependent on both voltage and angle of magnetization with easy axis $(G(V, \theta))$, integration over voltage gives the expression for the current through the device. 
For dynamic modeling, LLGS equation is employed to solve for magnetization vector at any moment. However, the coefficient used for the torque term is considered to be constant rather than $\theta$-dependent. In other words, $\theta$-dependency of the spin torque efficiency factor is ignored. Due to this, DM2 overestimates the switching time by $\% 5$ to $\% 10$. It also lacks thermal effect consideration and the stochastic nature of switching.

From the same group, there are two other papers published, [26] and [27], which describe the modeling of MTJ from magnetization and electrical aspects. [26] provides detailed information on sources of energy in the magnet such as magnetostatic energy. It then uses this information to form the $H_{e f f}$ in (3). Modified LLG equation is discussed to consider magnetic anisotropy and also coupling effects between ferromagnetic layers. It uses VHDL-AMS to capture the hysteresis and dynamic aspects of the magnetization. On the other hand, [27] provides details on static modeling and electrical behavior of MTJ. Several conductance models are discussed and the model uses a combination of some physics-based models such as Brinkman's model and Julliere's model.

The model in [28] follows almost the same approach. It is implemented in SPICE and follows Sun's model to solve LLG equation to derive the dynamic characteristic of an MTJ. For static characterization, Simmons tunnel current [29] is used, which deals with transmission and reflection probabilities as well as Fermi functions.

\section{Dynamic Model 3 (DM3)}

The model developed by K. Ono et al. [30] uses stochastic-LLG equation [31]. Voltage-dependent TMR is modeled using an exponential function as in (12). It also takes into account the temperature-dependency of TMR considering the fitting function as in (13) to model the relationship between polarization and temperature.

$$
\begin{aligned}
\operatorname{TMR}(T, V) & =\frac{2 P(T)^{2}}{1-P(T)^{2}} \exp \left(-V_{h}|V|\right) \\
P(T) & =P_{0}\left(1-a T^{\frac{3}{2}}\right)
\end{aligned}
$$

$V_{h}, P_{0}$, and $a$ are fitting parameters. Furthermore, thermal fluctuation is considered during the steps of the modeling.

This model is the most complete one among DM1 to DM3 as it uses LLG equation, which is more capable in capturing the transient behavior. Furthermore, voltage-dependency and temperature-dependency of parameters as well as stochastic nature of switching are considered.

\section{Dynamic Model 4 (DM4) in Verilog-A}

Conventional MTJs have their magnetization in-plane of the magnetic layer. Recently, new MTJs with magnetization perpendicular to the plane of magnetic layer have been developed. It was shown in [33] that in this structure the critical current can be reduced while maintaining high thermal stability.

While all the previous models target devices with in-plane magnetization, the dynamic model in [32] is for devices with perpendicular magnetization. It models the static behavior using Brinkman's model for conductance [20], as in [34], and employs voltage-dependent TMR ratio as in (8). Dynamic behavior is modeled according to the critical current calculation [35] along with the switching time derivation [36]. Furthermore, to capture thermal fluctuation effects, temperature value is randomly

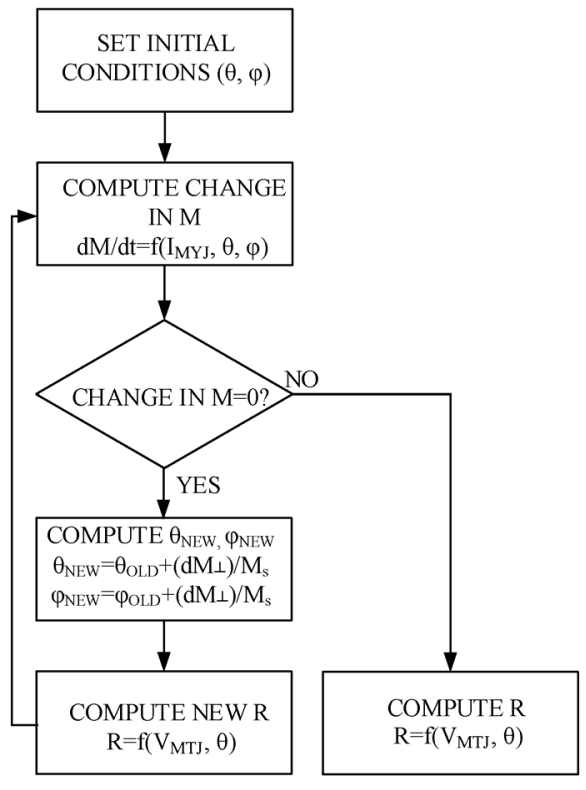

Fig. 6. Verilog-A model flowchart.

drawn from a uniform distribution. In order to verify the model, DC and transient simulations as well as statistical simulations are performed.

\section{E. Dynamic Model 5 (DM5) in Verilog-A}

As having an accurate model for MTJ is the basis for both device-level and circuit-level designs involving MTJ, we have implemented a dynamic model (accessible at [37]), which we describe in this section. We will use this model to compare and verify the above-mentioned models.

The model is implemented using Verilog-A and it consists of two components as done in DM2; MTJ resistance is used for static modeling and LLGS equation is deployed for dynamic characterization. However, unlike DM2, DM5 incorporates thermal fluctuation effects on switching.

Static behavior is predicted based on Julliere's model for conductance as in (2) along with voltage-dependent model of TMR ratio as in (8).

In order to characterize the dynamic behavior of magnetization vector, LLGS equation is implemented using 3 differential equations for $\mathrm{x}, \mathrm{y}$, and $\mathrm{z}$ components of the magnetization vector. Unlike DM2, $\theta$-dependency of the coefficient of torque term is considered (this eliminates $\% 5$ to $\% 10$ over-estimation of switching time). The Verilog-A code follows the simplified algorithm shown in Fig. 6.

This model uses physics-based equations. While voltage-dependency of TMR is captured, voltage-dependency of $\mathrm{G}$ as in (7) is not considered in this model to make it faster and less complex. DM2 considers both and integrates the small-signal conductance over voltage to get the current. Our model updates the resistance value according to corresponding $\theta$ and uses Ohm's law to get the voltage using the current and the resistance.

Employing the LLGS equation directly not only predicts the magnetization vector at any moment, but also eliminates the approximations made in the approach based on (5) and (6). In addition, the approach based on LLGS does not require any assumption on the amplitude of the current pulse. However, LLGS does not capture the effects of thermal fluctuation. Thermal fluctuation can affect the average precession motion as well as initial 


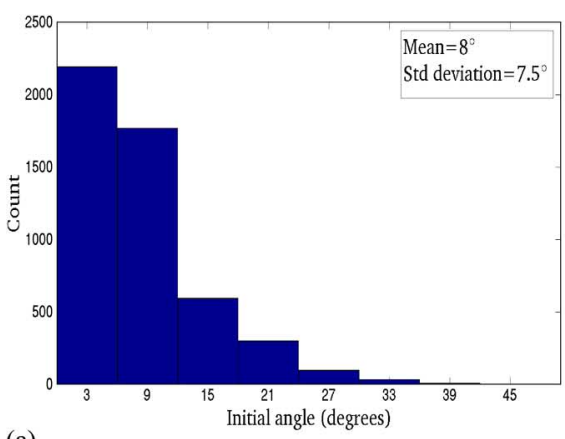

(a)

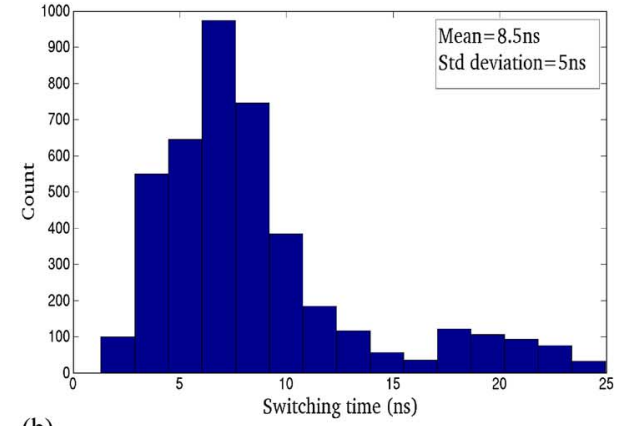

(b)

Fig. 7. (a) Distribution of initial angle of magnetization, (b) Distribution of switching time.

angle of magnetization, which is randomly distributed and plays a role in switching time [38]. To account for this, we randomized the initial state of the magnetization vector (initial $\theta$ ). For this, we chose a random Gaussian distribution [5] with a variance of

$$
\left\langle\theta_{0}^{2}\right\rangle=\frac{k_{b} T}{M_{s} V \mu_{0} H_{k}}
$$

in which $T$ is the temperature, $k_{b}$ is the Boltzmann constant, $M_{s}$ is the saturation magnetization, $V$ is the volume, $\mu_{0}$ is the permeability, and $H_{k}$ is the anisotropy constant. At room temperature and with typical device parameters that we used (i.e. $\mathrm{M}_{\mathrm{s}}=1050, \mathrm{H}_{\mathrm{K}}=250 \mathrm{Oe}$ ), standard deviation of $\theta_{0}$ is about $12^{\circ}$. As an example, Fig. 7(a) illustrates the distribution of initial angle for a set of 5000 data points. For this set, the mean is $8^{\circ}$ and standard deviation is $7.5^{\circ}$. Fig. 7(b) shows the distribution of switching time when a random initial angle is used. The mean of the switching time is $8.5 \mathrm{~ns}$ and its standard deviation is $5 \mathrm{~ns}$.

Note that the lower initial angles ( 0 to 6 degrees for example) result in a wider span of switching time (more than $18 \mathrm{~ns}$ ), whereas the higher initial angles (such as 10 to 40 degrees) result in a tighter span of switching time (less than $5 \mathrm{~ns}$ ). Due to this nonlinear relationship between the switching time and the initial angle, a linear mapping of initial angle bins and the switching time bins is not possible. For example, the last eleven bins of switching time histogram (switching time between $7 \mathrm{~ns}$ and 25 ns) correspond to the first bin of initial angle histogram (angles less than $6^{\circ}$ ).

Furthermore, $H_{\text {eff }}(\theta)$ is modified to model the effects of temperature on switching. This is done by adding a $H_{\text {fluctuation }}$ term (dependent on temperature) to $H_{\text {eff }}(\theta)$ of (3) [38].

For validation purposes, we compare in Fig. 8(a) the simulated switching time versus the MTJ current as obtained by DM5 against the corresponding one obtained by using (6). The simulation results (which are shown for $\theta_{0}=17^{\circ}$ as an example) are consistent with those based on (6).

Furthermore, for a fixed applied current of $\mathrm{I}=200 \mathrm{uA}$, the switching time as a function of the initial angle of magnetization in Fig. 8(b) are in agreement with the theoretical results based on (6).

To confirm that DM5 is accurately following (3) and (4) during transient, we compare its transient response against that of a straight-forward implementation in MATLAB. To this end, we apply a current pulse with 0.5 ns time constant to the MTJ, and predict both the trajectory of the magnetization

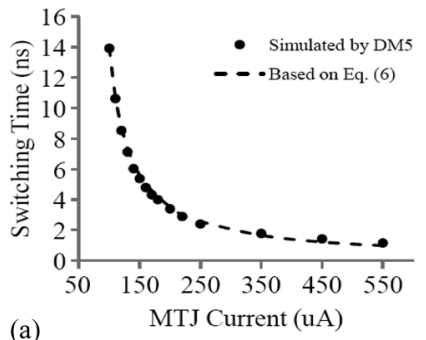

(a)

Fig. 8. (a) Switching time vs. applied current through MTJ (for $\theta_{0}=17^{\circ}$ ), (b) Switching time vs. initial angle of magnetization (for $\mathrm{I}=200 \mathbf{u A}$ ).

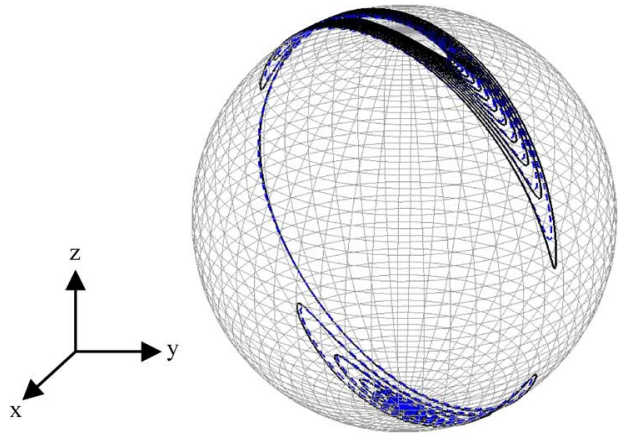

Fig. 9. Switching pattern of magnetization vector from $+z$ to $-z$ direction, solid black line is obtained using MATLAB, dashed blue line is by DM5.

vector and the magnetization as a function of time, using DM5 and MATLAB. While it takes two hours for MATLAB code to solve (3) and (4), it takes less than a minute for SPECTRE to run the Verilog-A code of DM5. Fig. 9 shows the switching trajectories as predicted by MATLAB (solid black line) and DM5 (dashed blue line), which are in close agreement. For the magnetization vector to switch from $+\mathrm{z}$ direction to $-\mathrm{z}$ direction, it moves up and down along ellipsoidal contours and as soon as it passes the easy plane of $\mathrm{x}-\mathrm{y}$, it damps toward $-\mathrm{z}$ direction. Note that the initial state is $(0,0.3, \operatorname{sqrt}(1-0.3 * 0.3)$ rather than $+z(0,0,1)$. This gives the initial magnetization some deviation from parallel state and produces a non-zero spin torque. In simulation, this is done by setting the initial state to the corresponding value (which could be either a fixed value or a random one). In reality, the tilt occurs due to thermal fluctuation and the switching starts from a random initial angle.

Fig. 10 compares the z-component of magnetization vector as a function of time, as predicted by MATLAB (solid black line) and by DM5 (blue dashed line). The two results are in close agreement. 


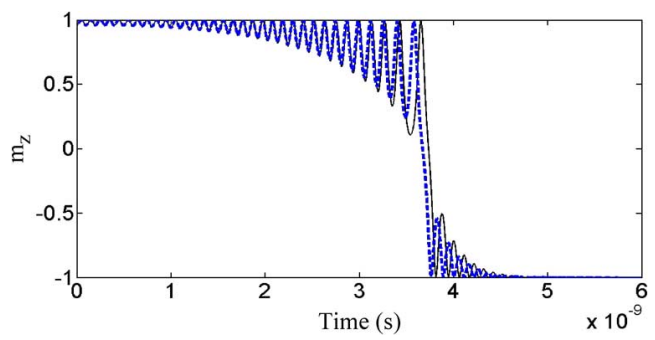

Fig. 10. Z-component of magnetization for non-zero time constant current pulse, obtained from MATLAB (solid black line) vs. DM5 results (dashed blue line)

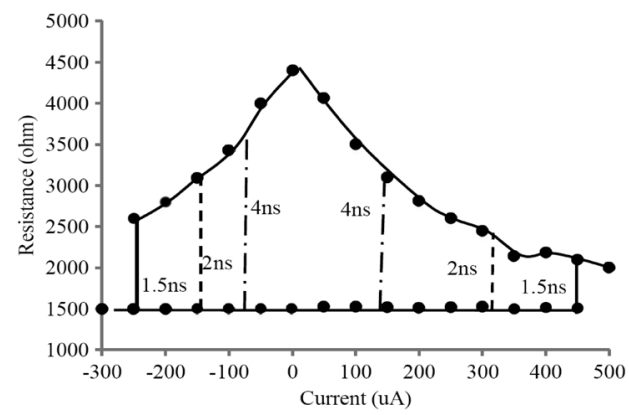

Fig. 11. Hysteresis resistance of MTJ versus applied current.

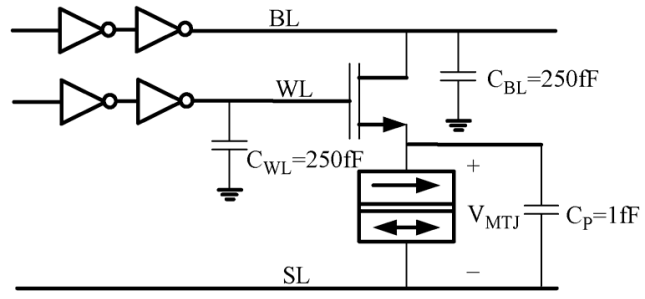

Fig. 12. System-level simulation of 1T-1MTJ structure.

In the remainder of this section, we employ DM5 along with SPECTRE for circuit simulations including MTJs. The hysteresis behavior of the MTJ resistance versus the applied current is shown in Fig. 11. For fixed pulsewidths (i.e. 1.5 ns, 2 $\mathrm{ns}, 4 \mathrm{~ns}$ ), the applied current is swept for different values and switching is observed at corresponding switching currents (the higher the pulsewidth, the lower the switching current). The resistance value is calculated by dividing the voltage across the MTJ by the applied current. Fig. 11 illustrates the constant parallel resistance and the voltage-dependent anti-parallel resistance of MTJ. Also, note that the three sets of the switching points are shown for three different pulsewidths of 1.5, 2, and 4 ns.

Fig. 12 shows a one-transistor, one MTJ (1T-1MTJ) cell structure along with its bitline (BL) and wordline (WL) drivers. We assume the parasitic capacitances of $250 \mathrm{fF}, 250 \mathrm{fF}$, and 1 $\mathrm{fF}$ for $\mathrm{WL}, \mathrm{BL}$, and the storage node, respectively. While $B L$ is raised to VDD and $S L$ is set to GND, the $W L$ signal is driven to VDD to write to the MTJ.

Simulated waveforms, including $B L, W L, V_{M T J}, I$, and the z-component of the magnetization vector $\left(m_{z}\right)$ are illustrated in Fig. 13. During the time period that BL and WL are high and the current passes through the transistor and the MTJ, the magnetization vector switches from $+\mathrm{z}$ to $-\mathrm{z}$ direction (from parallel to anti-parallel). Due to increase in resistance of the
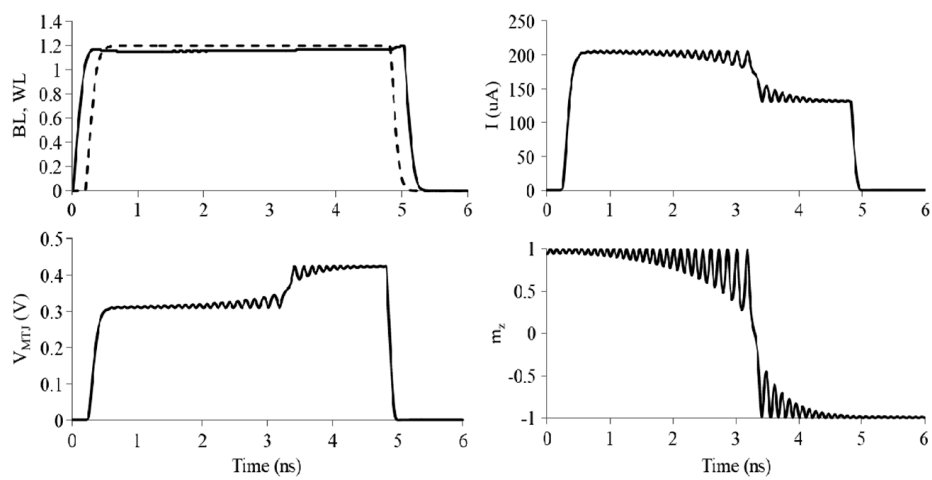

Fig. 13. 1T-1MTJ simulation results.

TABLE II

COMPARISON OF DYNAMIC MODELS

\begin{tabular}{|c|c|c|c|c|}
\hline Models & Implement. & LLGS & $\begin{array}{c}\text { Thermal } \\
\text { fluctuations }\end{array}$ & $\begin{array}{c}\text { Simulation } \\
\text { time }\end{array}$ \\
\hline DM1 [24] & Verilog-A & No & Yes & Shorter \\
\hline DM2 [25] & VHDL-AMS & Yes & No & Longer \\
\hline DM3 [30] & Not specified & Yes & Yes & Longer \\
\hline DM4 [32] & Verilog-A & No & Yes & Shorter \\
\hline DM5 (This work) & Verilog-A & Yes & Yes & Longer \\
\hline
\end{tabular}

MTJ (from $R_{p}$ to $R_{A P}$ ), the voltage across the MTJ increases, while its current decreases.

\section{COMParative Study of DyNAMic Models}

DM1 to DM5, as discussed in Section VI, are compared in Table II in terms of their methods of implementation, modeling bases, simulation time, and whether or not they consider thermal fluctuation effects.

As discussed in Section III-B, there are two approaches to characterize the MTJ's dynamic behavior. While DM2, DM3, and DM5 use (3) and (4) (by deploying LLGS equation), DM1 and DM4 follow (5) and (6) (by calculating the switching time).

The approach based on LLGS equation monitors the magnetization vector at any moment and fully captures its transient behavior. On the other hand, the second approach uses (5) and (6) to predict the switching time. Even though these equations are based on physics of MTJ and are derived using LLGS equation, there are several approximations and assumptions involved to simplify the analysis [39].

One of the assumptions is the small initial deviation of magnetization vector from the easy axis (preferred direction in steady state). However, there are situations, such as the one depicted in Fig. 14, in which the initial angle at the transition of the applied current is not small. For example, the initial deviations at the second rising edges of the applied currents are $10^{\circ}$ and $21^{\circ}$, respectively. These two different initial deviations result in $1.7 \mathrm{~ns}$ and $0.83 \mathrm{~ns}$ of switching time. While pulse\#1 gives enough time for the magnetization vector to relax and get back to the easy plane, pulse $\# 2$ turns on again before reaching the steady state. Note that due to the limited accuracy of the simulations, even with zero $\mathrm{x}$ and $\mathrm{y}$ components, the z-component may not settle on $-\mathrm{z}$ because of roundings that happen during each step of solving the differential LLGS equations. 


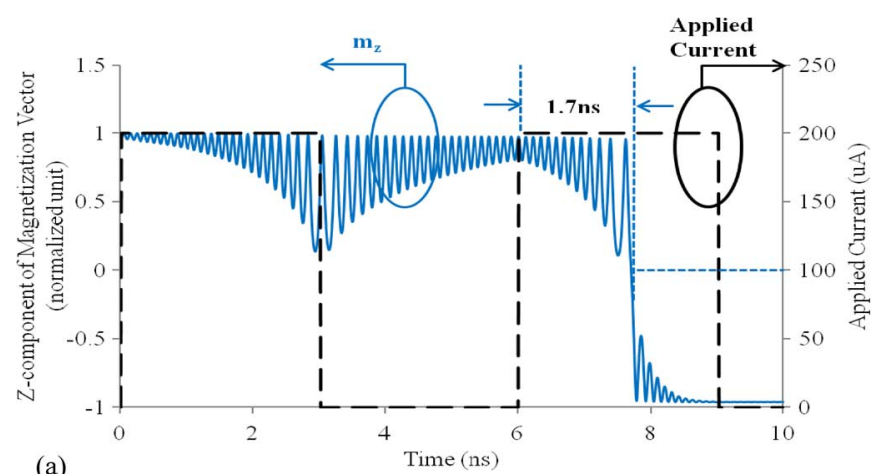

(a)

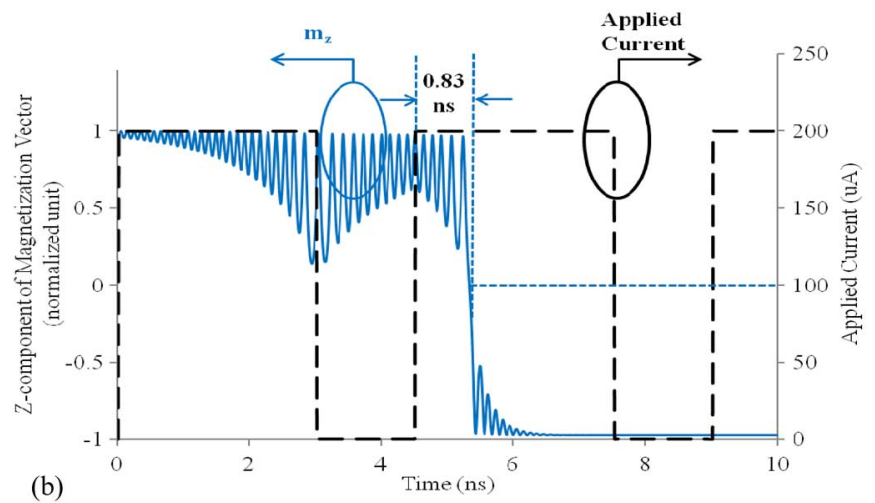

Fig. 14. Z-component of magnetization vector (a) for pulse \#1, (b) for pulse \#2.

This results in deviation of the magnitude of the magnetization vector from unity.

The other assumption made is constant amplitude for the applied current pulse. This assumption is not valid in the presence of any parasitic capacitance that gives rise to a non-zero time constant. To show the difference in switching time, two current pulses, one with a zero time constant and one with a non-zero time constant, are applied through the MTJ and the results are shown in Fig. 15. The zero time constant pulse makes the MTJ switch about $1 \mathrm{~ns}$ faster than the other pulse. To account for this, [40] suggests updating the switching time and the switching probability at every time step. It uses NS-SPICE to implement an algorithm to predict switching time.

Even though LLGS equation is more capable of predicting the magnetization vector at any moment for any kind of input, it is unable to model the effects of thermal fluctuation on switching. Therefore, $\mathrm{H}_{\mathrm{eff}}$ should be modified accordingly in order to account for thermal effects. The model in [41] uses a stochastic LLG equation and adds a temperature-dependent fluctuating term to the torque. Furthermore, it uses heat diffusion equation to solve for temperature. Also, it uses temperature-dependent MTJ resistance. All these are solved self-consistently using SPICE components.

On the other hand, the second approach based on (5) and (6) considers temperature, and the switching due to thermal fluctuation (even when the current is more than critical current), but it does not monitor the magnetization vector continuously and it is based on assumptions, such as rectangular current pulse, and approximations like small deviation from easy axis. Therefore, the best dynamic modeling would be based on LLGS equation, while incorporating thermal fluctuation effects and variations.
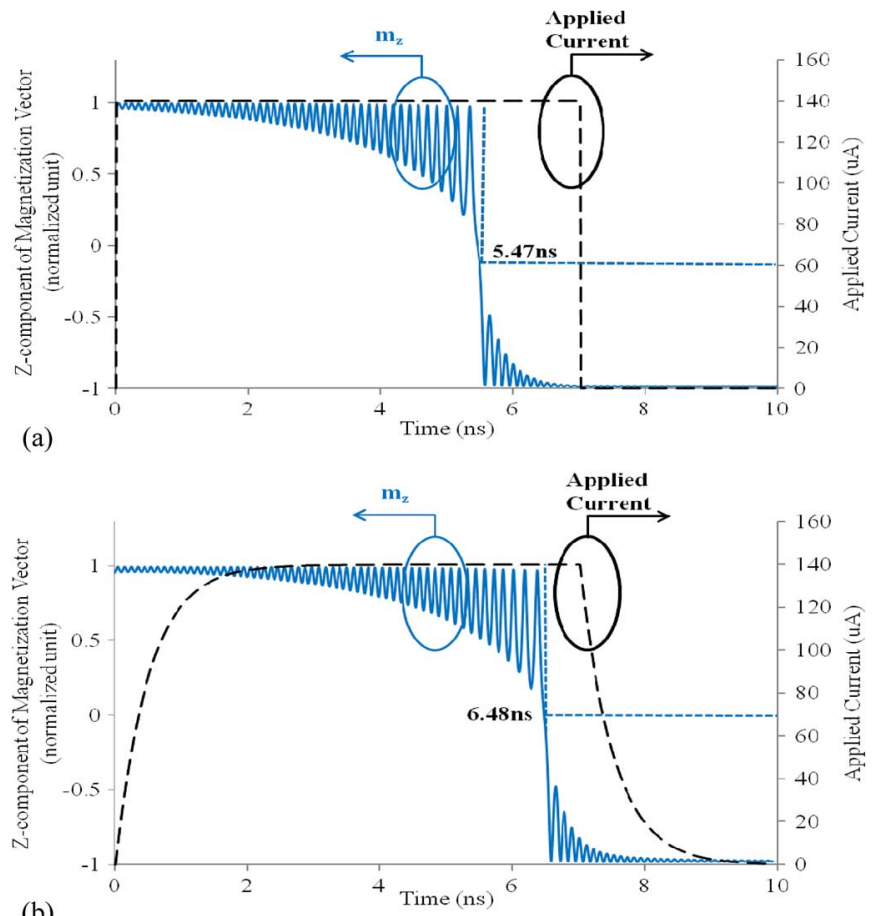

(b)

Fig. 15. Z-component of magnetization vector (a) for ideal current pulse, (b) for non-ideal current pulse.

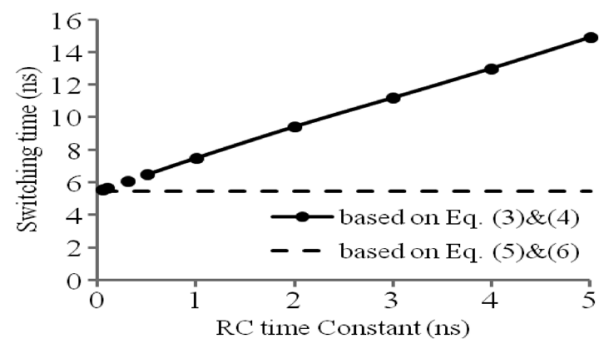

Fig. 16. Switching time vs. time constant of applied current pulse.

The tradeoff for predicting the transient behavior and for higher accuracy is longer simulation time and higher computational cost. Models based on LLGS equations take longer to simulate while the models based on (5) and (6) are faster but ignore the transient behavior. Fig. 16illustrates the predicted switching time for applied current pulses with different time constants. While the approach based on (3) and (4) predict a linear increase in switching time as a function of time constant, the approach based on (5) and (6) predicts a constant switching time as it ignores the time constant. These simulation results are obtained by using DM5.

Alternative MTJ structures such as thermally assisted switching MTJ or MTJ with perpendicular polarizer are modeled in [42], [43] in similar ways as discussed above.

\section{CONCLUSION}

We classified the MTJ models as static and dynamic and compared them against each other in terms of accuracy, implementation, and simulation time. Among the static models, SM3 is the most accurate as it uses physical equations rather than curve fitting to obtain results. Among the dynamic models, DM3 and DM5 (proposed in this paper) are based on LLGS equation and as such avoid using any approximation in predicting the transient behavior of the MTJ. In addition, they include the effects 
of variations such as thermal fluctuations. We used DM5 in conjunction with SPECTRE to study the transient behavior of an MTJ in response to a current pulse with non-zero time constant. For a range of $0 \mathrm{~ns}$ to $5 \mathrm{~ns}$, we showed that the switching time linearly increases with the time constant. These results are in sharp contrast with those predicted by (5) and (6) where the time constant is ignored in switching time predictions.

\section{ACKNOWLEDGMENT}

The authors would like to thank the Canadian Microelectronic Corporation $(\mathrm{CMC})$ for providing the $\mathrm{CAD}$ tools.

\section{REFERENCES}

[1] A. Driskill-Smith, D. Apalkov, V. Nikitin, X. Tang, S. Watts, D. Lottis, K. Moon, A. Khvalkovskiy, R. Kawakami, X. Luo, A. Ong, E. Chen, and M. M. Krounbi, "Latest advances and roadmap for in-plane and perpendicular STT-RAM," in Proc. 3rd IEEE Int. Memory Workshop $(I M W)$, May 22-25, 2011, pp. 1-3.

[2] K. C. Chun, H. Zhao, J. D. Harms, T.-H. Kim, J.-P. Wang, and C. H. Kim, "A scaling roadmap and performance evaluation of in-plane and perpendicular MTJ based STT-MRAMs for high-density cache memory," IEEE J. Solid-State Circuits, vol. 48, no. 2, pp. 598, 610, Feb. 2013.

[3] D. D. Tang and Y. Lee, Magnetic Memory: Fundamentals and Technology. Cambridge, U.K.: Cambridge University Press, 2010.

[4] B. Behin-Aein, A. Sarkar, S. Srinivasan, and S. Datta, "Switching energy-delay of all spin logic devices," Appl. Phys. Lett., vol. 98, pp. 123510-1-123510-3, Aug. 2011.

[5] S. Manipatruni, D. E. Nikonov, and I. A. Young, "Modeling and design of spintronic integrated circuits," IEEE Trans. Circuits Syst., Reg. Papers, vol. 59, no. 12, pp. 2801-2814, Dec. 2012.

[6] J.-G. Zhu, "Magnetoresistive random access memory: The path to competitiveness and scalability," Proc. IEEE, vol. 96, no. 11, pp. 1786, 1798, Nov. 2008

[7] C. Augustine, N. N. Mojumder, X. Fong, S. H. Choday, S. P. Park, and K. Roy, "Spin-transfer torque MRAMs for low power memories: Perspective and prospective," IEEE Sensors J., vol. 12, no. 4, pp. 756-766, April 2012.

[8] R. Dorrance, F. Ren, Y. Toriyama, A. A. Hafez, C.-K. K. Yang, and D. Markovic, "Scalability and design-space analysis of a 1T-1MTJ memory cell for STT-RAMs," IEEE Trans. Electron Devices, vol. 59, no. 4 , pp. 878-887, 2012

[9] [Online]. Available: http://bwrcs.eecs.berkeley.edu/Classes/IcBook/SPICE/

[10] [Online]. Available: http://www.cadence.com/products/rf/spectre_circuit/pages/default.aspx

[11] J. C. Slonczewski, "Current-driven excitation of magnetic multilayers," J. Magnetism Magnetic Mater., vol. 159, p. L1, 1996.

[12] M. Julliere, "Tunneling between ferromagnetic films," Phys. Lett., vol. 54A, pp. 225-226, 1975.

[13] L. D. Landau and E. M. Lifshitz, "Theory of the dispersion of magnetic permeability in ferromagnetic bodies," Phys. Z. Sowietunion, vol. 8, p. $153,1935$.

[14] T. L. Gilbert, "A Lagrangian formulation of the gyromagnetic equation of the magnetic field," Phys. Rev., vol. 100, p. 1243, 1995.

[15] Z. Diao, Z. Li, S. Wang, Y. Ding, A. Panchula, E. Chen, L. Wang, and Y. Huai, "Spin-transfer torque switching in magnetic tunnel junctions and spin-transfer torque random access memory," J. Phys. Condens. Matter, vol. 19, no. 16, April 2007

[16] R. H. Koch, J. A. Katine, and J. Z. Sun, "Time-resolved reversal of spin-transfer switching in a nanomagnet," Phys. Rev. Lett., vol. 92, no. 8, p. $088302,2004$.

[17] S. S. Mukherjee and S. K. Kurinec, "A stable SPICE macro-model for magnetic tunnel junctions for applications in memory and logic circuits," Trans. Magn., vol. 45, no. 9, pp. 3260-3268, Sep. 2009.

[18] J. D. Harms, F. Ebrahimi, X. Yao, and J.-P. Wang, "SPICE macromodel of spin-torque-transfer-operated magnetic tunnel junctions," IEEE Trans. Electron Devices, vol. 57, no. 6, pp. 1425-1430, Jun. 2010.

[19] W. Zhao, E. Belhaire, Q. Mistral, C. Chapped, V. Javerliac, B. Dieny, and E. Nicolle, "Macro-model of spin-transfer torque based magnetic tunnel junction device for hybrid magnetic-CMOS design," in Proc. IEEE Int. Behavioral Modeling Simulation Workshop, 2006, pp. 40-43.
[20] W. F. Brinkman, R. C. Dynes, and J. M. Rowell, "Tunneling conductance of assymetrical barriers," J. Appl. Phys., vol. 41, no. 5, pp. $1915-1921,1970$.

[21] L. Fei-fei, Z.-Z. Li, M.-W. Xiao, J. Du, W. Xu, A. Hu, and J. Q. Xiao, "Bias dependent tunneling in ferromagnetic junctions and inversion of the tunneling magnetoresistance from a quantum mechanical point of view," J. Appl. Phys., vol. 95, no. 11, pp. 7243-7245, 2004.

[22] J. C. Slonczewski, "Currents, torques, polarization factors in magnetic tunnel junctions," J. Phys. Rev. B, vol. 71, p. 024411, 2005.

[23] M. Hosomi, H. Yamagishi, T. Yamamoto, K. Bessho, Y. Higo, K. Yamane, H. Yamada, M. Shoji, H. Hachino, C. Fukumoto, H. Nagao, and H. Kano, "A novel nonvolatile memory with spin-torque transfer magnetization switching: Spin-ram," in IEDM Tech. Dig., Dec. 2005, pp. $459-462$.

[24] L. B. Faber, W. Zhao, J. O. Klein, T. Devolder, and C. Chappert, "Dynamic compact model of spin-transfer torque based Magnetic Tunnel Junction (MTJ)," in Proc. DTIS Conf., 2009, pp. 130-135.

[25] M. Madec, J. B. Kammerer, F. Pregaldiny, L. Hebrard, and C. Lallement, "Compact modeling of magnetic tunnel junction," in NEWCASTAISA, 2008, pp. 229-232.

[26] J.-B. Kammerer, M. Madec, and L. Hébrard, "Compact modeling of a magnetic tunnel junction-Part I: Dynamic magnetization model," IEEE Trans. Electron Devices, vol. 57, no. 6, pp. 1408-1415, Jun 2010.

[27] M. Madec, J.-B. Kammerer, and L. Hébrard, "Compact modeling of a magnetic tunnel junction-Part II: Tunneling current model," IEEE Trans. Electron Devices, vol. 57, no. 6, pp. 1416-1424, Jun. 2010.

[28] A. Nigam, K. Munira, A. Ghosh, S. Wolf, E. Chen, and M. R. Stan, "Self consistent parameterized physical MTJ compact model for STTRAM," in Proc. Int. Semiconductor Conf. (CAS), Oct. 11-13, 2010, pp. 423-426.

[29] J. G. Simmons, "Electrical tunnel effect between dissimilar electrodes separated by a thin insulating film," J. Appl. Phys., vol. 34, no. 2581, 1963.

[30] K. Ono, T. Kawahara, R. Takemura, K. Miura, H. Yamamoto, M. Yamanouchi, J. Hayakawa, K. Ito, H. Takahashi, S. Ikeda, H. Hasegawa, H. Matsuoka, and H. Ohno, "A disturbance-free read scheme and a compact stochastic-spin-dynamics-based MTJ circuit model for Gb-scale SPRAM," in Proc. IEDM, 2009, pp. 1-4.

[31] Z. Li and S. Zhang, "Thermally assisted magnetization reversal in the presence of spin-transfer torque," Phys. Rev. B, vol. 69, p. 134416 , 2004.

[32] Y. Zhang, W. Zhao, Y. Lakys, J. Klein, J. Kim, D. Ravelosona, and C. Chappert, "Compact modeling of perpendicular-anisotropy $\mathrm{CoFeB} / \mathrm{MgO}$ magnetic tunnel junctions," IEEE Trans. Electron Devices, vol. 59, no. 3, pp. 819-826, Mar. 2012.

[33] S. Mangin, D. Ravelosona, J. A. Katine, M. J. Carey, B. D. Terris, and E. E. Fullerton, "Current-induced magnetization reversal in nanopillars with perpendicular anisotropy," Nature Mater., vol. 5, pp. 210-215, Mar. 2006.

[34] W. S. Zhao, J. Duval, J. O. Klein, and C. Chappert, "A compact model for magnetic tunnel junction (MTJ) switched by thermally assisted spin transfer torque (TAS+STT)," Nanoscale Res. Lett., vol. 6, no. 1, p. 368 , 2011.

[35] S. Ikeda, K. Miura, H. Yamamoto, K. Mizunuma, H. D. Gan, M. Endo, S. Kanai, H. Hayakawa, F. Matsukura, and H. Ohno, "A perpendicular anisotropy $\mathrm{CoFeB}-\mathrm{MgO}$ magnetic tunnel junction," Nature Mater. vol. 9, no. 9, pp. 721-724, 2010 .

[36] D. C. Worledge, G. Hu, D. W. Abraham, J. Z. Sun, P. L. Trouilloud, J. Nowak, S. Brown, M. C. Gaidis, E. J. Osullivan, and R. P. Robertazzi, "Spin torque switching of perpendicular $\mathrm{Ta}|\mathrm{CoFeB}| \mathrm{MgO}$-based magnetic tunnel junctions," Appl. Phys. Lett., vol. 98, no. 2, pp. 0225011-022501-3, Jan. 2011.

[37] [Online]. Available: http://www.eecg.utoronto.ca/ ali/mram.html

[38] J. Z. Sun, "Spin angular momentum transfer in current-perpendicular nanomagnetic junctions," IBM J. Res. Develop., vol. 50, no. 1, pp. 81, 100, Jan. 2006

[39] J. Z. Sun, "Spin-current interaction with a monodomain magnetic body: A model study," Phys. Rev. B, vol. 62, no. 1, pp. 570-578, 2000.

[40] N. Sakimura, R. Nebashi, Y. Tsuji, H. Honjo, T. Sugibayashi, H. Koike, T. Ohsawa, S. Fukami, T. Hanyu, H. Ohno, and T. Endoh, "High-speed simulator including accurate MTJ models for spintronics integrated circuit design," in Proc. 2012 IEEE ISCAS, May 2012, pp. 1971, 1974.

[41] G. Panagopoulos, C. Augustine, and K. Roy, "A framework for simulating hybrid MTJ/CMOS circuits: Atoms to system approach," in Proc. Design, Automation \& Test Eur. Conf. Exhibition (DATE), March 2012, pp. 1443, 1446. 
[42] M. El Baraji, V. Javerliac, W. Guo, G. Prenat, and B. Dieny, "Dynamic compact model of thermally assisted switching magnetic tunnel junctions," J. Appl. Phys., vol. 106, no. 12, p. 123906-123906-6, 2009.

[43] A. Mejdoubi, G. Prenat, and B. Dieny, "A compact model of precessional spin-transfer switching for MTJ with a perpendicular polarizer," in Proc. MIEL, 2012, pp. 225-228.

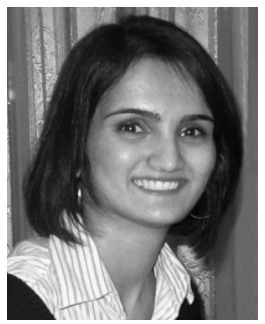

Aynaz Vatankhahghadim received the B.Sc. degree from Sharif University of Technology, Tehran, Iran, in 2008 and the M.A.Sc. degree from University of Waterloo, Waterloo, Canada, in 2010 both in electrical engineering. She is currently working towards the $\mathrm{Ph} . \mathrm{D}$. degree in electrical engineering at University of Toronto.

Her research interests include spintronic circuits focusing on modeling, device-level design and circuit-level design of STT-MRAM.

Ms. Vatankhahghadim has held Ontario Graduate Scholarship for Science and Technology (OGSST), Rogers scholarship and the University of Waterloo scholarship.

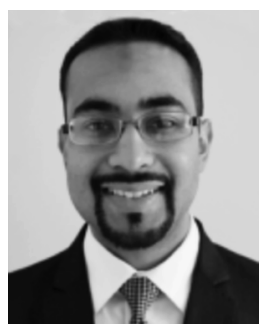

Safeen Huda (S'13) received the B.A.Sc. and M.A.Sc. degrees in electrical engineering from the University of Toronto, Toronto, ON, Canada in 2009 and 2012, respectively. He is currently working toward the Ph.D. degree in computer engineering at the University of Toronto.

His research interests include spintronic circuits, low power circuit design, development of CAD tools for digital circuit optimization, and FPGAs.

Mr. Huda has held the Natural Sciences and Engineering Research Council of Canada (NSERC), Canada Graduate Scholarship and the University of Toronto Fellowship.

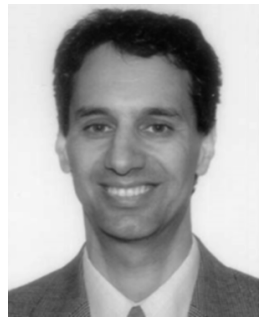

Ali Sheikholeslami (S'98-M'99-SM'02) received the B.Sc. degree from Shiraz University, Iran, in 1990 and the M.A.Sc. and Ph.D. degrees from the University of Toronto, Canada, in 1994 and 1999, respectively, all in electrical and computer engineering.

In 1999, he joined the Department of Electrical and Computer Engineering, University of Toronto, where he is currently a Professor. His research interests are in the areas of analog and digital integrated circuits, high-speed signaling, and VLSI memory design. He has collaborated with industry on various research projects, including work with Fujitsu Labs of Japan and America. He was a visiting researcher with Fujitsu Labs in 2005-2006, and with Analog Devices in 2012-2013.

Dr. Sheikholeslami has received the Best Professor of the Year Award four times (in 2000,2002, 2005, and 2007) by the popular vote of the undergraduate students in the Department of Electrical and Computer Engineering, University of Toronto. He received the 2005-2006 Early Career Teaching Award and the 2010 Faculty Teaching Award, both from the Faculty of Applied Science and Engineering at the University of Toronto, in "Recognition of Superb Accomplishment in Teaching". He served on the Memory, Technology Directions, and Wireline Subcommittees of the ISSCC in 2001-2004, 2002-2005, and 2007-2013, respectively. He currently serves on the executive committee of the same conference as its Educational Events Chair, and on the editorial board of the Solid-State Circuits Magazine as an Associate Editor. He was an Associate Editor for the IEEE TRANSACTIONS ON CIRCUITS AND SYSTEMS-I: REGULAR PAPERS for 2010-2012. He was the program chair for the 2004 IEEE ISMVL held in Toronto, Canada. He is a registered professional engineer in the province of Ontario, Canada. 